\title{
Intoxicação espontânea por Brachiaria híbrida cv mulato I em ovinos e caprinos e experimental por Brachiaria híbrida cv mulato II em ovinos ${ }^{1}$
}

\author{
Daiane Ogliari ${ }^{2}$, Franciéli A. Molossi² ${ }^{2}$ Thaiza Savaris², Nathalia S. Wicpolt ${ }^{2}$, \\ Ildo Zancan $^{3}$ e Aldo Gava ${ }^{2 *}$
}

\begin{abstract}
Ogliari D., Molossi F.A., Savaris T., Wicpolt N.S., Zancan I. \& Gava A. 2018. [Spontaneous poisoning by Brachiaria hybrid cv. mulato I in goats and sheep and experimental poisoning of Brachiaria hybrid cv. mulato II in sheep.] Intoxicação espontânea por Brachiaria híbrida cv mulato I em ovinos e caprinos e experimental por Brachiaria híbrida cv mulato II em ovinos. Pesquisa Veterinária Brasileira 38(2):229-233. Laboratório de Patologia Animal, Centro de Ciências Agroveterinárias, Universidade do Estado de Santa Catarina, Lages, SC 88520-000, Brazil. E-mail: aldo.gava@udesc.br

Described is the epidemiology, clinical signs and lesions of a disease that leads to photosensitization and death in sheep and goats. Spontaneous disease occurred in the municipality of Abelardo Luz, SC. From a flock of 350 sheep and 278 goats grazing Brachiaria hybrid cv. mulato I, 27 animals got sick, which 17 died and 10 recovered after being removed from the pasture. The disease was characterized by tearing, hyperemia, swelling of face, ears and eyelid followed by crusting, serous nasal discharge, ulcers on the cornea and sclera, and conjunctiva slightly yellowish. Six sheep and eleven goats were necropsied on the farm. The macroscopic lesions were brownish liver, distended gallbladder and lumpy bile, brownish kidneys with red dots on surface. Through microscopy was observed mild to moderate lesions in liver mainly characterized by swelling and vacuolation of hepatocytes, megalocytosis, periportal fibrosis, biliary hyperplasia; small clusters of macrophages and infiltration of eosinophils, neutrophils and macrophages were present in the periportal area. Crystal negative images were detected within bile ducts. Kidney tubules were dilated ranging from mild to severe with glomerular tuff contraction and distension of Bowman space. Four sheep experimentally fed Brachiaria hybrid cv. mulato II ad libitum for 90 days showed no clinical changes.

INDEX TERMS: Poisoning plants, Brachiaria hybrid cv. mulato, plant poisoning, photosensitization, sheep, goats, toxicoses.
\end{abstract}

RESUMO.- Descreve-se a epidemiologia, sinais clínicos e lesões de uma enfermidade em ovinos e caprinos que cursa com fotossensibilização e morte. A doença espontânea ocorreu no município de Abelardo Luz, SC. De um rebanho de

\footnotetext{
${ }^{1}$ Recebido em 27 de abril de 2016.

Aceito para publicação em 7 de novembro de 2016.

Dissertação de Mestrado do primeiro autor, concluída no ano de 2016 na Universidade do Estado de Santa Cantarina.

${ }^{2}$ Laboratório de Patologia Animal, Centro de Ciências Agroveterinárias (CAV), Universidade do Estado de Santa Catarina (UDESC), Av. Luiz de Camões 2090, Bairro Conta Dinheiro, Lages, SC 88520-000, Brasil. *Autor para correspondência: aldo.gava@udesc.br

${ }^{3}$ Veterinário autônomo, Rua João Smedt 1262, Abelardo Luz, SC 89830000, Brasil.
}

350 ovinos e 278 caprinos, de corte, mantidos em pastagem de Brachiaria híbrida cv mulato I, adoeceram 27 animais, dos quais, 17 morreram e 10 recuperaram-se após terem sido removidos do local. A enfermidade caracterizou-se por lacrimejamento, hiperemia, edema das faces, das orelhas e das pálpebras seguidas de formação de crostas, corrimento nasal seroso, ulcerações na córnea e esclera e conjuntiva levemente amarelada. Seis ovinos e onze caprinos foram necropsiados na propriedade. As lesões macroscópicas observadas foram fígado de coloração castanha, vesícula biliar com conteúdo aumentado e bile grumosa, rins acastanhados com pontos vermelhos na superfície. Na avaliação microscopia foram observadas no fígado, lesões de intensidade leve a moderada caracterizadas principalmente por tumefação e ou, vacuolização 
de hepatócitos, megalocitose, fibrose periportal, proliferação biliar, pequenos aglomerados de macrófagos, aleatórios e infiltrado de eosinófilos, neutrófilos e macrófagos na periferia do lóbulo. Na luz de ductos biliares encontravam-se imagens negativas de cristais. Nos rins havia dilatação de túbulos variando de leve a acentuada, contração de tufo glomerular e distensão do espaço de Bowman. Quatro ovinos receberam experimentalmente Brachiaria híbrida cv mulato II ad libitum pelo período de 90 dias e não manifestaram alterações clinicas.

TERMOS DE INDEXAÇÃO: Plantas tóxicas, Brachiaria híbrida cV mulato, intoxicação por plantas, fotossensibilização, ovinos, caprinos, toxicoses.

\section{INTRODUÇÃO}

Pastagens formadas por Brachiaria spp. têm sido um obstáculo na criação de ovinos, caprinos e bovinos por causar intoxicação hepatógena, levando os animais à fotossensibilização, com diminuição da produção e morte. A ocorrência deste problema vem sendo observada desde a introdução de Brachiaria spp. no país (Andrade et al. 1975, Santos Junior 2008, Tokarnia et al. 2012). Os ovinos são tidos como uma das espécies mais sensíveis aos efeitos tóxicos da Brachiaria spp (Opasina 1985, Castro et al. 2007, Macedo et al. 2008).

Na literatura não há relatos da ocorrência de intoxicação por Brachiaria híbrida cV mulato em animais. No entanto, um surto de intoxicação por esta gramínea foi diagnosticado em ovinos e caprinos, no município de Abelardo Luz, Santa Catarina.

O objetivo do presente estudo foi avaliar os aspectos epidemiológicos, clínicos e patológicos de um surto de fotossensibilização por Brachiaria híbrida cv mulato I em ovinos e caprinos e testar experimentalmente o potencial tóxico da Brachiaria híbrida cv mulato II em ovinos.

\section{MATERIAL E MÉTODOS}

Doença espontânea. Efetuou-se levantamento sobre a ocorrência de doença fotossensibilizante que afetou ovinos e caprinos no município de Abelardo Luz, Oeste de Santa Catarina. Seis ovinos e onze caprinos acometidos pela enfermidade foram necropsiados e as amostras de tecidos foram coletadas e fixadas em formalina $10 \%$, para posterior realização de exame histológico no Laboratório de Patologia Animal da Universidade do Estado de Santa Catarina.

Estudo experimental. Para experimentação sementes de Brachiaria híbrida cv mulato II foram plantadas no mês de novembro de 2014, em um piquete no setor de Patologia Animal CAV/UDESC, e no início de fevereiro de 2015 a planta era cortada diariamente e fornecida ad libitum, durante 90 dias, para quatro ovelhas, fêmeas adultas, da mesma procedência, com idades semelhantes (4 anos) e sem contato prévio com Brachiaria spp. As ovelhas foram alojadasno setor de ovinocultura do CAV/UDESC, mantidas em baia de piso ripado de aproximadamente $20 \mathrm{~m}^{2}$, e num período aproximado de 5 horas diárias tinham acesso a luz solar em piquete anexo ao aprisco. Ao final do experimento as 4 ovelhas foram eutanasiadas para necropsia e amostras de todas as vísceras foram coletadas em formalina 10\%, para exame histológico.

Também foram avaliados históricos e efetuadas visitas a propriedade no municipio de Tunápolis - SC, onde bovinos pastejavam em $B$. híbrida cv mulato II.
Avaliações clínicas. Para a intoxicação experimental os animais foram pesados e submetidos a exames clínicos diários, sendo avaliados a frequência respiratória e cardíaca, temperatura retal, cor das mucosas, além da observação do comportamento. Para avaliação de possíveis alterações hematológicas, foram efetuadas coletas quinzenais de sangue da jugular para para a realização de hemogramas e a dosagem das enzimas hepáticas: Gama Glutamil Transferase (GGT), Aspartato Aminotransferase (AST), Fosfatase Alcalina (FA), bilirrubina. Na execução do experimento foi seguido procedimento analisado e aprovado pelo Comitê de Ética em Experimentação Animal da Universidade do Estado de Santa Catarina (CETEA/UDESC), protocolo número 1.04.15.

\section{RESULTADOS}

Doença espontânea. Sinais de fotossensibilização foram observados em alguns animais de um rebanho de 350 ovinos e 278 caprinos, de corte, no município de Abelardo Luz - SC, após um período aproximado de 60 dias de pastoreio exclusivamente em pastagem de Brachiaria híbrida cv mulato I (Fig.1). A enfermidade se caracterizou por lacrimejamento, hiperemia, aumento de volume das faces, das orelhas e das pálpebras seguidas de formação de crostas, corrimento seroso pelas narinas, opacidade e ulceração na córnea e a esclera e conjuntiva estavam levemente amareladas (Fig.2 e 3). Adoeceram 27 animais dos quais, 17 morreram e 10 recuperaram-se após terem sido removidos do local.

As lesões macroscópicas observadas foram fígado de coloração castanha, vesícula biliar com conteúdo aumentado e bile grumosa (Fig.4), rins acastanhados com pontos vermelhos na superfície. As principais lesões microscópicas foram observadas no fígado com intensidade leve a moderada e consistiram de tumefação, vacuolização e megalocitose de hepatócitos, fibrose periportal, retenção biliar, proliferação de ductos biliares, pequenos aglomerados de macrófagos (Fig.5) e na periferia de alguns lóbulos havia infiltrado de eosinófilos, neutrófilos e alguns macrófagos. Na luz de alguns ductos biliares também foram observadas imagens negativas de cristais (Fig.6). Nos rins havia dilatação de túbulos leve a acentuada e os glomérulos tinham contração do tufo glomerular e distensão do espaço de Bowman. 0 ovino que manifestou opacidade ocular, tinha necrose da córnea acompanhado por

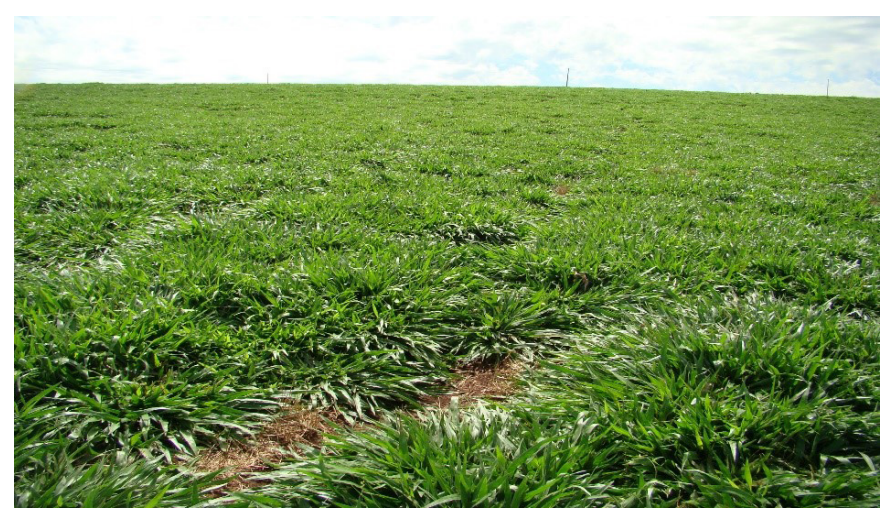

Fig.1. Pastagem de Brachiaria híbrida cv mulato I, no município de Abelardo Luz/SC. 


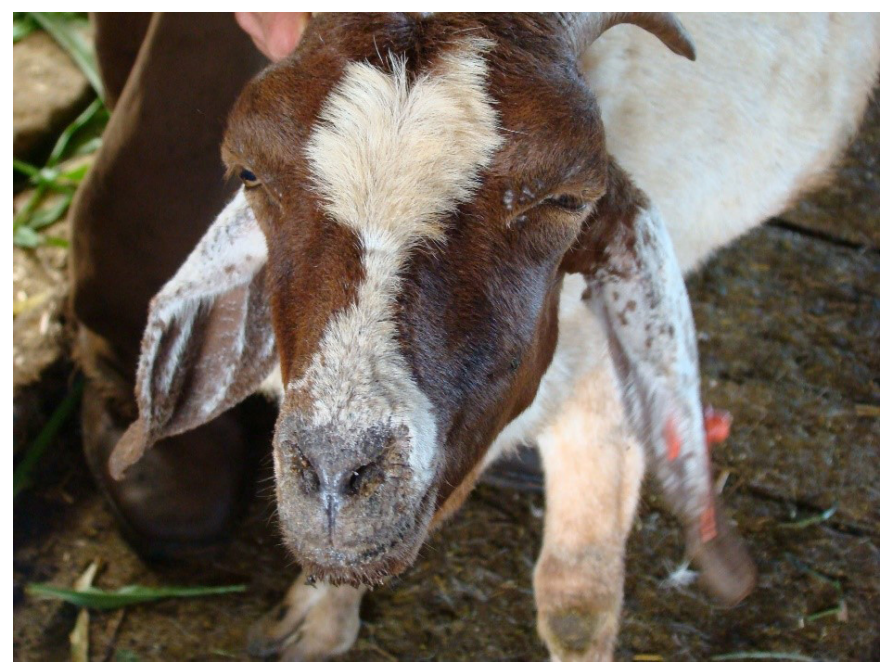

Fig.2. Intoxicação espontânea por Brachiaria híbrida cv mulato I. Caprino com face e orelhas edemaciadas e crostas nas narinas.

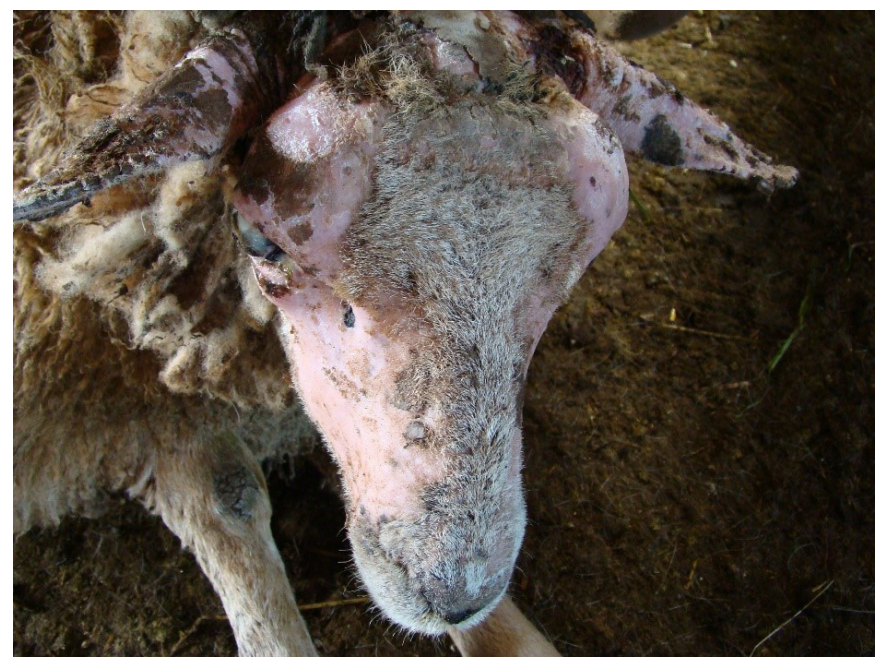

Fig.3. Intoxicação espontânea por Brachiaria híbrida cv mulato I. Ovino com áreas hiperêmicas e alopécicas, crostas nas faces e narinas e opacidade de córnea.

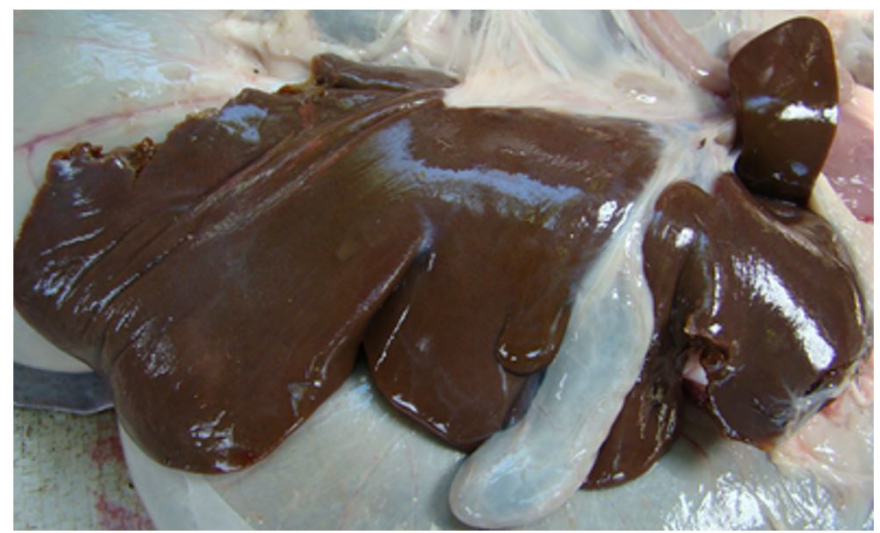

Fig.4. Intoxicação espontânea por Brachiaria híbrida cv mulato I em caprino. Fígado castanho e vesícula biliar distendida.

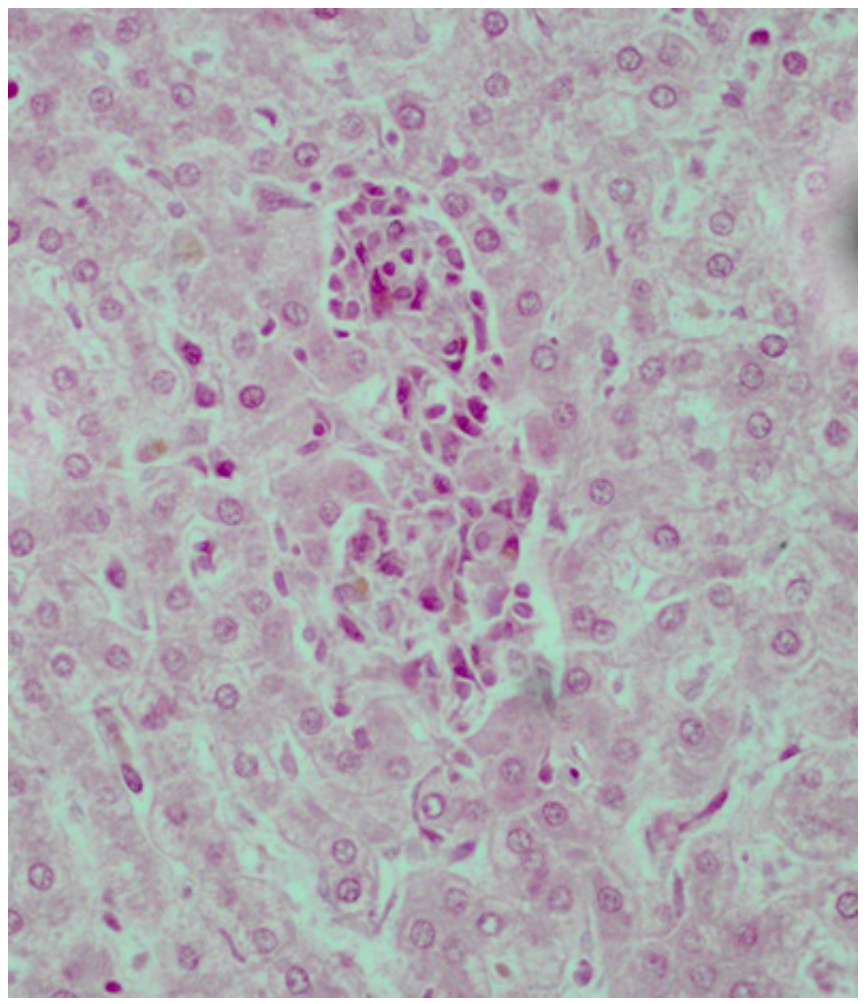

Fig.5. Intoxicação espontânea por Brachiaria híbrida cv mulato I em ovino. Parênquima hepático com acúmulo de macrófagos. HE, obj.40x.

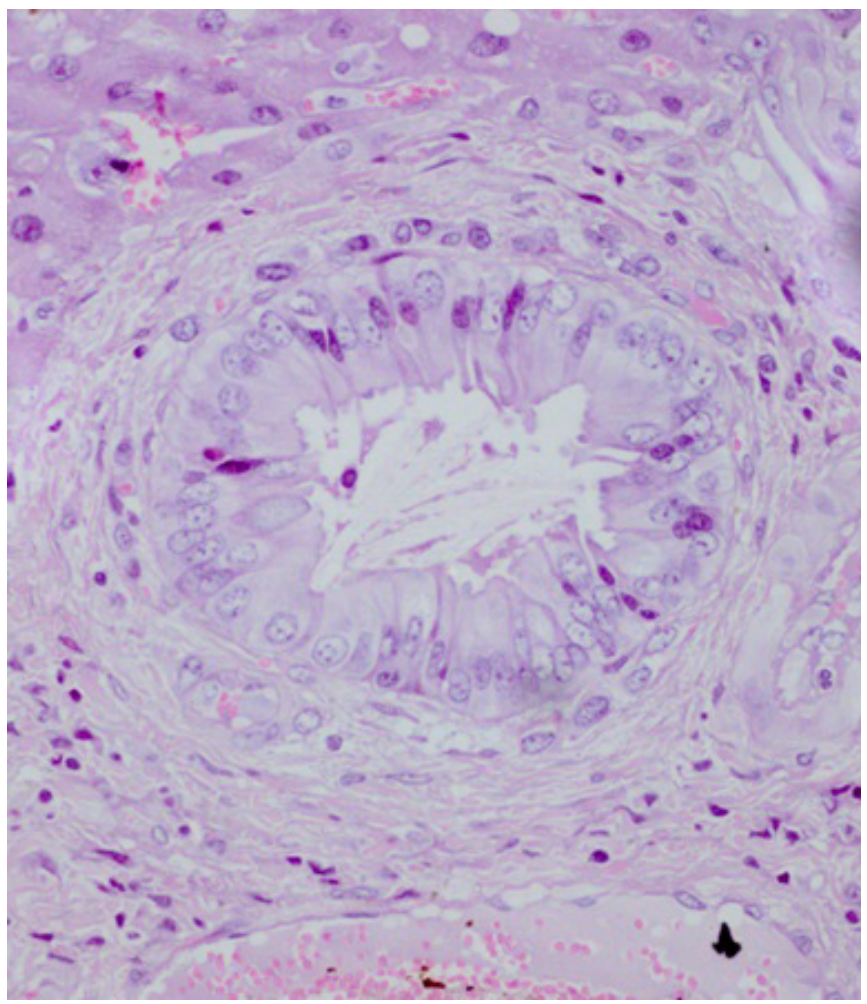

Fig.6. Intoxicação espontânea por Brachiaria híbrida cv mulato I. em caprino. Parênquima hepático com imagens negativas de cristais na luz de ductos biliares. HE, obj.40x. 
edema acentuado, infiltrado de neutrófilos e deposição de fibrina e restos celulares.

Após a confirmação do diagnóstico de intoxicação por $B$. híbrida cv mulato I, o produtor foi orientado a dividir os animais em dois lotes. Cada lote permaneceria por no máximo 30 dias sobre $B$. híbrida cv mulato I e 30 dias em outra área constituída por outras gramíneas, principalmente Brachiaria plantaginea (Capim-doce, papuã) e a doença desapareceu. Nos anos seguintes o produtor restringiu a adubação e usou a área em consorciação de $B$. híbrida cv mulato I com Cynodon nlemfuensis Vanderyst (Tifton 85) e a doença não ocorreu.

Intoxicação experimental. Os quatro ovinos que receberam B. híbrida cv mulato II ad libitum pelo período de 90 dias, não manifestaram alterações clinicas. Uma ovelha (no74) pariu no vigésimo dia de experimento e o cordeiro permaneceu com a mãe durante todo o período de experimento e também não apresentou sinais de intoxicação. Na necropsia, não foram observadas lesões macroscópicas e microscópica.

Em uma propriedade no município de Tunápolis/SC, um lote de 30 vacas Jersey alimentadas exclusivamente $\operatorname{com} B$. híbrida cV mulato II e complementação com ração comercial foram acompanhadas por dois anos e também não manifestaram alterações clínicas.

As amostras de sangue coletadas para hemograma, bilirrubina e a dosagem de enzimas hepáticas não mostraram alterações.

\section{DISCUSSÃO}

O surto de fotossensibilização espontânea observado em ovinos e caprinos no Município de Abelardo Luz foi relacionado a ingestão prolongada de $B$. híbrida cv mulato $I$. No período, a pastagem apresentava-se vigorosa, pois o proprietário utilizava dejetos de suínos na adubação da gramínea e esta era a única fonte de alimentação para os ovinos e caprinos. 0 crescimento vigoroso da Brachiaria parece ser um importante fator para o desenvolvimento da enfermidade. Isto está de acordo com Peixoto (2008, dados não publicados) citado por Tokarnia et al. (2012) que atribuem maior toxicidade da Brachiaria spp., quando esta tem crescimento vigoroso e cresce em áreas mais úmidas. Lemos et al. (1997), também atribui o bom desenvolvimento da B. decumbens, como fator importante para a produção de fotossensibilização em bovinos.

O tempo necessário para o aparecimento dos sinais clínicos após o início da ingestão de Brachiaria spp. é bastante variável: de 7 a 38 dias (Porto et al. 2013), 15 dias a mais de 12 meses (Mustafa et al. 2012), 20 a 62 dias (Saturnino et al. 2010), 30 dias (Macedo et al. 2008), 14 dias (Castro et al. 2007) e 10 dias (Opasina 1985). No presente estudo, os ovinos e caprinos manifestaram alterações clínicas 60 dias após início do pastoreio sobre Brachiaria híbrida cv mulato I. O período maior de pastoreio necessário para o surgimento da doença, pode indicar que $B$. híbrida cv mulato I é menos tóxica que as demais espécies de Brachiaria.

Na propriedade onde ocorreu o surto havia consorciação de ovinos e caprinos, sendo que os caprinos adoeceram em maior número. Lemos et al. (1998) observaram surto de fotossensibilização em caprinos que se alimentavam de Brachiaria decumbens, no entanto, ovinos mantidos na mesma área não apresentaram a doença. Macedo et al. (2008) e Opasina (1985), ao avaliarem surtos de fotossensibilização em ovinos e caprinos alimentados com Brachiaria brizantha e $B$. decumbens, respectivamente, observaram que os ovinos adoeceram em maior número. Esta variação de sensibilidade às Brachiaria spp., entre as diferentes espécies de animais, sugere que a maior sensibilidade não está relacionada a espécie animal, mas sim a outros fatores como adaptação dos animais e manejo.

No surto observado em Abelardo Luz, o proprietário foi orientado para intercalar períodos de pastoreios entre $B$. híbrida cv mulato e outro tipos de pastagem e diminuir a adubação para que a pastagem se tornasse menos vigorosa. As alterações de manejo com redução de tempo de pastoreio sobre a $B$. híbrida cv mulato I e o uso de consorciação com outras gramíneas foram medidas bem sucedidas para evitar a enfermidade. Este manejo e ou, a redução na adubação do solo, permitem o uso racional de consumo da planta, evitando assim prejuízos econômicos pela fotossensibilização produzida pela $B$. híbrida cv mulato I.

As manifestações de fotossensibilização, os achados de necropsia e as principais lesões microscópicas são observações já citadas por Graydon et al. (1991), Lemos et al. (1996, 1998), Cruz et al. (2000), Driemeier et al. (2002), Albernaz et al. (2010), Saturnino et al. (2010), Mustafa et al. (2012) e Porto et al. (2013), em intoxicações por B. decumbens e B. brizantha. No entanto, a maioria dos autores descrevem macrófagos espumosos no fígado. Nos ovinos do presente estudo, raramente estas células foram observadas e não foram encontradas nos caprinos. Lemos et al. (1998), também não descrevem estas células em caprinos intoxicados por $B$. decumbens.

Nos experimentos realizados com $B$. híbrida cV mulato II, os animais não apresentaram alterações clínicas decorrentes da ingestão de $B$. híbrida cv mulato II no período experimental. Um cordeiro, nascido de uma ovelha em experimentação também não mostrou sinais de fotossensibilização. Em bovinos mantidos em pastagem desta gramínea no município de Tunápolis/SC, acompanhados por dois anos também não produziram alterações clinicas. Este fato pode indicar que possivelmente esta gramínea não possui potencial tóxico para produzir fotossensibilização em animais. No entanto, devemos levar em consideração que o presente experimento foi efetuado com a planta com vigor moderado de crescimento.

Lemos et al. (1997), Santos et al. (2008) e Mustafa (2009) sugerem que a menor ocorrência de casos de intoxicação em ovinos adultos, seja devido a alguma forma de adaptação ou resistência dos ruminantes à pastagem. Também citam a herdabilidade como fator a ser considerado e pode estar relacionado aos mecanismos de susceptibilidade e resistência de ovinos à intoxicação. Os ovinos usados neste experimento eram da raça Lacaune e nunca tiveram contato prévio com Brachiaria.

De acordo com os resultados do presente experimento, a utilização de $B$. híbrida cv mulato II mostra-se como alternativa viável e estratégica para formação de pastagens, umas vez que não mostrou-se tóxica a ovinos em um período de 90 dias, no entanto, esta gramínea deve ser manejada e adubada adequadamente. 


\section{CONCLUSÕES}

Brachiaria híbrida cv mulato I, cultivada em solo bem adubado pode produzir fotossensibilização e morte em ovinos e caprinos.

Brachiaria híbrida cv mulato II, não produziu experimentalmente alterações clinicas em ovinos.

A utilização de Brachiaria híbrida cv mulato II, por 90 dias pode ser alternativa viável e estratégica para formação de pastagens em substituição a outras espécies de Brachiaria comprovadamente tóxicas.

\section{REFERÊNCIAS}

Albernaz T.T., Silveira J.A.S., Silva N.S., Oliveira C.H.S., Belo Reis A.S., Oliveira C.M.C., Duarte M.D. \& Barbosa J.D. 2010. Fotossensibilização em ovinos associada à ingestão de Brachiaria brizantha no Estado do Pará. Pesq. Vet. Bras. 30(9):741-748. http://dx.doi.org/10.1590/S0100-736X2010000900006.

Andrade S.A., Nothenberg M.S., Retz L. \& Bueno P.C. 1975. Estudos sobre cobaias alimentadas com Brachiaria sp. Arq. Inst. Biológico, São Paulo 42:243-246.

Castro M.B., Moscardini A.R.C., Reckviegel G.C., Novaes E.P.F., Mustafa V.S., Guedes K.M.R., Paludo G.R., Borges J.R. \& Riet-Correa F. 2007. Susceptibilidade de ovinos a intoxicação por Brachiaria decumbens. V Congreso Latinoamericano de Especialistas en Pequeños Ruminantes y Camélidos Sudamericanos, Buenos Aires, Argentina. Disponível em <http://www.aleprycs.net/ documents/21709/30558/Castro,+intoxicaci\%C3\%B3n.pdf> Acesso em 27 set. 2015.

Cruz C., Driemeier D., Pires V.S., Colodel E.M., Taketa A.T.C. \& Schenkel E. 2000. Isolation of steroidal saponins implicated in experimentally induced cholangiopathy of sheep grazing Brachiaria decumbens in Brazil. Vet. Hum. Toxicol. 42(3):142-145. PMid:10839316.

Driemeier D., Colodel E.M., Seitz A.L., Barros S.S. \& Cruz L.E.F. 2002. Study of experimentally induced lesions in sheep by grazing Brachiaria decumbens. Toxicon 40(7):1027-1031. PMid:12076657. http://dx.doi.org/10.1016/ S0041-0101(01)00276-8.

Graydon R.J., Hamid H., Zahari P. \& Gardiner C. 1991. Photosensitization and crystal associated cholangiohepatopathy in sheep grazing Brachiaria decumbens. Aust Vet. J. 68(7):234-236. PMid:1929990. http://dx.doi. org/10.1111/j.1751-0813.1991.tb03214.x.

Lemos R.A.A., Ferreira L.C.L., Silva S.M., Nakazato L. \& Salvador S.C. 1996. Fotossensibilização e colangiopatia associada a cristais em ovinos em pastagem com Brachiaria decumbens. Ciência Rural 26(1):109-113. http:// dx.doi.org/10.1590/S0103-84781996000100020.

Lemos R.A.A., Salvador S.C. \& Nakazato L. 1997. Photosensitization and Crystal associated cholangiohepatopathy in cattle grazing Brachiaria decumbens in Brazil. Vet. Hum. Toxicol. 39(6):376-377. PMid:9397512.
Lemos R.A.A., Nakazato L., Herrero Junior G.O., Silveira A.C. \& Porfírio L.C. 1998. Fotossensibilização associada a cristais em caprinos mantidos sob pastagens de Brachiaria decumbens no Mato Grosso do Sul. Ciência Rural 28(3):507-510. http://dx.doi.org/10.1590/S0103-84781998000300026.

Macedo J.T.S.A., Riet-Correa F., Dantas A.F.M. \& Simões S.V.D. 2008. Doenças da pele em caprinos e ovinos no Semi-árido brasileiro. Pesq. Vet. Bras. 28(12):633-642.http://dx.doi.org/10.1590/S0100-736X2008001200013.

Mustafa V.S. 2009. Caracterização da intoxicação natural por Brachiaria spp. em ovinos no Brasil Central. Dissertação de Mestrado em Saúde Animal, Faculdade de Agronomia e Medicina Veterinária, Universidade de Brasília, Brasília, DF. Disponível em <http://repositorio.unb.br/ bitstream/10482/7192/1/2009_VanessadaSilvaMustafa\%5b1\%5d.pdf> Acesso em 22 set 2014. 63p.

Mustafa V.S., Moscardini A.R.C., Borges J.R.J., Reckziegel G.C., Riet-Correa F. \& Castro M.B. 2012. Intoxicação natural por Brachiaria spp. em ovinos no Brasil Central. Pesq. Vet. Bras. 32(12):1272-1280. http://dx.doi. org/10.1590/S0100-736X2012001200010.

Opasina B.A. 1985. Photosensitization jaundice syndryme in West African dwarf sheep and goats grazed on Brachiaria decumbens. Trop. Grasslands 19:120-123.

Peixoto P.V. 2008. Departamento de Nutrição Animal e Pastagens. UFRRJ, Seropédica, RJ. Dados Não Publicados.

Porto M.R., Saturnino K.C., Lima E.M.M., Lee S.T., Lemos R.A.A., MarcolongoPereira C., Riet-Correa F. \& Castro M.B. 2013. Avaliação da exposição solar na intoxicação experimental por Brachiaria decumbens em ovinos. Pesq. Vet. Bras. 33(8):1009-1015. http://dx.doi.org/10.1590/S0100736X2013000800011.

Santos Júnior H.L. 2008. Estudo de toxicidade de diferentes estágios de crescimento de Brachiaria decumbens em ovinos. Dissertação de Mestrado em Saúde Animal, Faculdade de Agronomia e Medicina Veterinária, Universidade de Brasília,B, Brasília. Disponível em <http://repositorio. unb.br/bitstream/10482/4633/1/2009_HelvecioLealSantosJunior.pdf> Acesso em 17 out 2014.70 p.

Santos J.C.A., Riet-Correa F., Simões S.V. \& Barros C.S.L. 2008. Patogênese, Sinais clínicos e patologia das doenças causadas por plantas hepatotóxicas em ruminantes e equinos no Brasil. Pesq. Vet. Bras. 28(1):1-14. http:// dx.doi.org/10.1590/S0100-736X2008000100001.

Saturnino K.C., Mariani T.M., Barbosa-Ferreira M., Brum K.B., Fernandes C.E.S. \& Lemos R.A.A. 2010. Intoxicação por Brachiaria decumbens em ovinos confinados. Pesq. Vet. Bras. 30(3):195-202. http://dx.doi.org/10.1590/ S0100-736X2010000300002.

Tokarnia C.H., Brito M.F., Barbosa J.D., Peixoto P.V. \& Döbereiner J. 2012. Plantas Tóxicas do Brasil. Helianthus, Rio de Janeiro. 566 p. 